\title{
LA INSCRIPCIÓN DE LVPERCA \\ DEL TETRAPYLON DE CAPERA \\ (CÁPARRA, CÁCERES). \\ HALLAZGO Y CUESTIONES HISTORIOGRÁFICAS
}

\author{
LVPERCA'S INSCRIPTION \\ ON THE TETRAPYLON OF CAPERA (CÁPARRA, CÁCERES). \\ NEWS OF THE DISCOVERY AND HISTORIOGRAPHICAL ISSUES
}

\author{
Julio Esteban Ortega \\ Universidad de Extremadura \\ jesteban@unex.es
}

ORCID: 0000-0002-7628-8333

DOI: $10.1387 /$ veleia.18076

\begin{abstract}
Resumen: El tetrapylon de Capera es un monumento a la memoria de la familia de M. Fidius Macer. El reciente descubrimiento de la inscripción de la esposa de este magistrado, Iulia Luperca, que estuvo en el pilar izquierdo del lado suroeste del arco, supone un hallazgo importante para la arqueología de la ciudad de Capera. Desarrollamos en este trabajo el análisis epigráfico de la inscripción, así como las vicisitudes históricas de la misma durante los últimos siglos.

Palabras clave: Capera, Epigrafía Romana, Tetrapylon, Historiografía.

Abstract: The Capera tetrapylon is a monument to the memory of M. Fidius Macer's family. The recent discovery of this inscription related to the wife of this magistrate, Iulia Luperca, located on the left pillar of the southwest side of the arch, is an important discovery for the archaeology of the city of Capera. This paper offers an, epigraphic analysis of the inscription and comments on its historical vicissitudes over the past centuries.

Keywords: Capera, Roman Epigraphy, Tetrapylon, Historiography.
\end{abstract}

Recibido: 04-07-2016

Informado: 20-01-2017

Definitivo: 16-03-2017

Hasta las primeras excavaciones arqueológicas llevadas a cabo por Floriano Cumbreño en 1929 muy poco era lo que se conocía de la antigua ciudad romana de Capera (Floriano 1944, 270-286). A finales del siglo iII o en el IV de nuestra Era comenzó un lento pero inexorable proceso de deterioro del recinto urbano que terminó con el olvido más absoluto. Sus grandiosos edificios públicos se derrumbaron, las viviendas fueron abandonadas y sus habitantes buscaron nuevos horizontes. Solamente el majestuoso arco cuadrifonte que presidía la ciudad resistió los embates del tiempo. Los mármoles que revestían el foro fueron convertidos en cal y solo unas pocas piezas emblemáticas escaparon a la destrucción. Este fue el caso de algunas inscripciones que sustentaban los pedes- 
tales de estatuas o presidían los principales monumentos de la ciudad. Andando el tiempo, buena parte de ellas terminaron adornando los palacios renacentistas de los nobles que, siguiendo la nueva corriente humanista de la época, crearon auténticas colecciones de antigüedades romanas.

Una de estas piezas que salieron de las ruinas de Cáparra fue la inscripción que rotulaba el pedestal de estatua de Iulia Luperca, esposa del constructor del tetrapylon, M. Fidius Macer.

Mucho es lo que se ha especulado sobre este epígrafe, cuyas primeras referencias se remontan a textos manuscritos del siglo XVI. Ya en el siglo xIx, Hübner creía firmemente que las estatuas de la insigne pareja de notables caperenses descansaba sobre los pedestales del lado suroeste del arco cuadrifonte, ocupando la de Luperca el pedestal del pilar izquierdo del monumento. A este respecto señala el epigrafista alemán: Ad arcum Caperensem pertinuisse ignoraverunt Hispani inde a Castro (CIL II, 835).

Otros investigadores le siguieron en años sucesivos, aunque no faltan los que pusieron en duda tal hipótesis. Entre los primeros, García y Bellido (1972-1974, 63), quien se lamentaba de no conocer «las dimensiones de la piedra para asegurar donde se dice procede». Entre los segundos, Nünnerich-Asmus $(1996,26)$, que no aprecia espacio suficiente para la colocación de las estatuas.

¿Era realmente el pedestal izquierdo del arco el lugar que ocupaba la inscripción de Luperca? Las descripciones ofrecidas por quienes decían haberla visto eran poco o nada detalladas, por lo que difícilmente sirvieron para aventurar hipótesis acerca de su ubicación. Se necesitaba conocer las características de la pieza para poder dilucidar esta posibilidad.

Con el reciente descubrimiento del sillar con la inscripción de Iulia Luperca en uno de nuestros viajes epigráficos a la ciudad de Plasencia creemos ver cumplidos los deseos de cuantos coincidieron con Hübner y aliviados los temores de aquellos que rechazaron sus argumentos.

Desde hace algunos años vengo estudiando la epigrafía de la zona de Cáparra para la elaboración del corpus de inscripciones latinas de la provincia de Cáceres y mis desplazamientos a la capital del Jerte han sido frecuentes. En esta labor encontré siempre la compañía de mi buen amigo José Antonio Pajuelo Jiménez, perfecto conocedor de la zona y enamorado de la Historia de Plasencia ${ }^{1}$. En unos de estos viajes visitamos la Casa de los Vargas, posteriormente sede del Seminario Menor y actualmente residencia sacerdotal, donde tuvimos oportunidad de estudiar y fotografiar las cinco inscripciones que allí se conservaban. En este lugar, debajo de un banco a la entrada de la estancia, había un gran bloque de granito muy deteriorado del que se había desprendido la mayor parte de la capa exterior y donde se apreciaban restos de letras de lo que debió de ser en otro tiempo una inscripción monumental.

Poca información pude sacar en los primeros análisis de la misteriosa piedra. Estaba a punto de abandonar en mi empeño, cuando se me ocurrió cotejar los textos de las inscripciones desaparecidas que los autores antiguos habían visto en la casa del Deán con los pocos trazos que aún conservaba el bloque de granito. Cuál fue mi sorpresa cuando caí en la cuenta que estaba en presencia de la tan buscada inscripción de la esposa de M. Fidius Macer. El paso siguiente fue desplazarme a la ruinas de Cáparra y comparar las medidas de la piedra con las correspondientes a las del pedestal izquierdo del arco cuadrifonte. En líneas siguientes ofrezco el resultado de dichas investigaciones.

${ }^{1}$ Quiero expresar mi más profundo agradecimiento a José Antonio Pajuelo Jiménez por la inestimable ayuda prestada en todos los aspectos de la investigación. Él me facilitó los contactos y me acompañó siempre en los trabajos de campo. Su impagable labor ha contribuido de manera decisiva a que este trabajo viera la luz. Hago ex- tensivo este agradecimiento a D. Antonio Luis Galán, deán de la catedral de Plasencia, que amablemente me permitió el acceso a la inscripción e hizo posible la realización de este estudio. Y, por último, mi reconocimiento a los informantes externos de la revista, cuyos comentarios han contribuido a mejorar este trabajo. 
Comencé las pesquisas documentándome a partir de las fuentes consultadas por Hübner para la elaboración del CIL. Dichas fuentes me condujeron, como ya señalamos, a manuscritos del siglo XVI en los que se recogían las primeras referencias acerca de la inscripción. Posteriormente consulté los libros de viajes del XVIII y revisé las recopilaciones de inscripciones del XIX. Finamente, recabé las noticias e investigamos los autores del siglo xx que se refieren directa o indirectamente a ella. Los resultados fueron muy interesantes, como habrá ocasión de comprobar, pero antes analicemos los detalles morfológicos, el contenido epigráfico de la inscripción y su ubicación en el monumento.

\section{LA INSCRIPCIÓN}

Está elaborada sobre un bloque rectangular de granito anaranjado del lugar de $43 \times 131 \times 33 \mathrm{~cm}$. Se encuentra en avanzado estado de deterioro al haberse desprendido la mayor parte de la capa exterior que contenía el texto. En la parte superior se aprecian restos de una capa de cemento que recorre longitudinalmente el bloque. Solo se conservan algunas letras completas y trazos de otras, aunque en determinados lugares se observan las marcas, en forma de manchas con tonalidades más oscuras, dejadas por las filtraciones de humedad donde una vez estuvieron las desaparecidas letras. Este deterioro del granito puede verse perfectamente en muchos bloques del arco de los que se ha desprendido la fina capa del revestimiento superficial.

Los restos de cemento sugieren que en algún momento el sillar estuvo empotrado con el texto hacia el interior del muro. Todo parece indicar que tras su extracción se intentó limpiar y se desprendió buena parte de la capa que sustentaba el texto, aunque es muy posible también que el desprendimiento pudiera producirse en el momento mismo de la extracción.

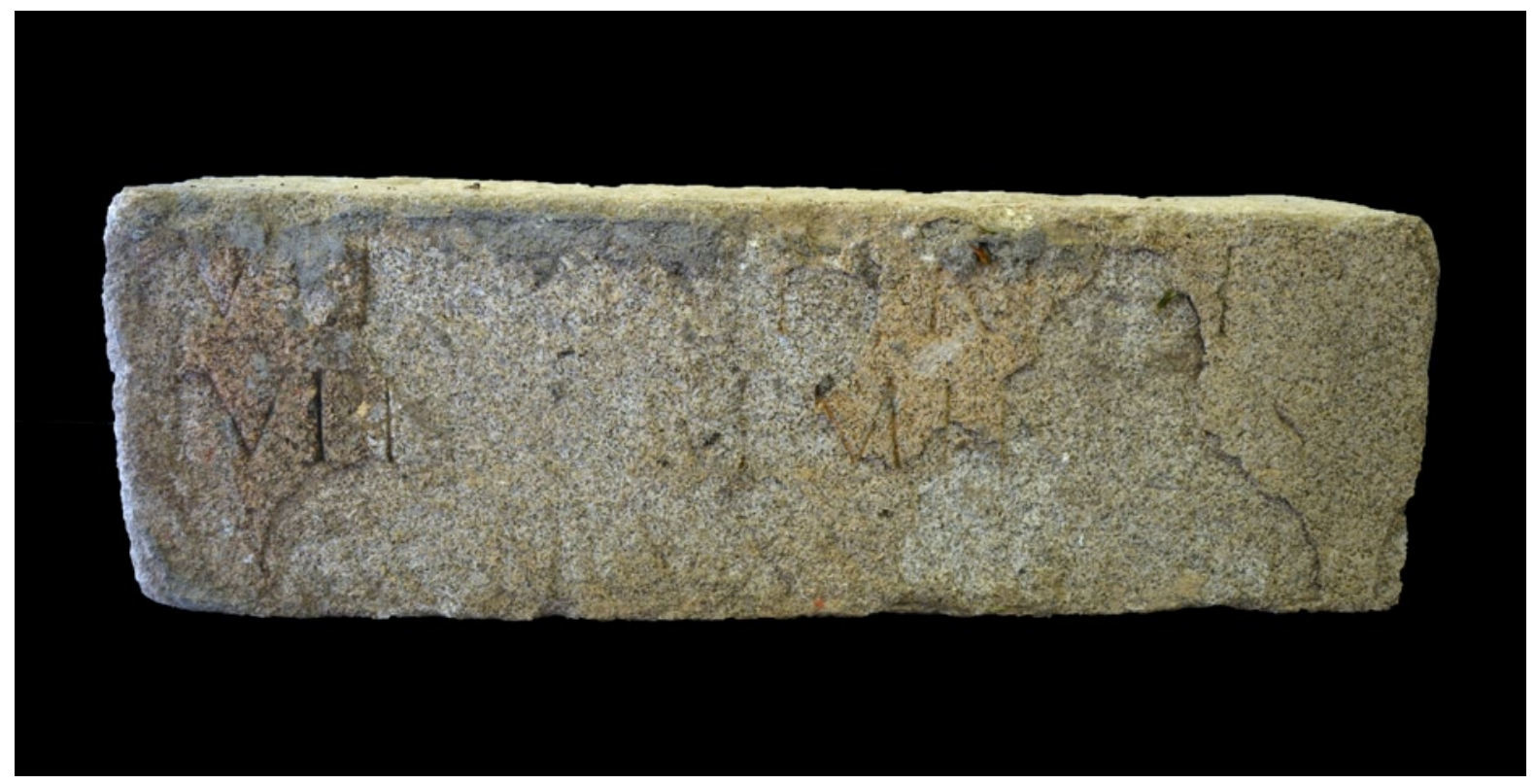

Figura I. Sillar con la inscripción de Luperca 


\title{
IVLIA]E IVPERCIS F LVPERCAE M FID]US $M A C E \mathbb{R}$ VXORI I I
}

Figura 2. Reconstrucción

Las letras de $9 \mathrm{~cm}$, con bisel triangular y pies bien marcados, son capitales cuadradas de época flavia, idénticas a las de la inscripción del otro pedestal del arco (fig. 1). El texto reza así (fig. 2)2

\author{
Iuliae Lupercif f(iliae) \\ Lupercae M(arcus) Fidius \\ Macer uxṣori p(oni) i(ussit)
}

Accursio, ms. 1550; Castro, RAH 9-6002-9; Toro, ms. 1573, fig. 22; Gruterus, 1602, 6; Muratori, 1740, n. ${ }^{\circ}$ 1356, 5; Mayans 1746, 4; Morales y Tercero, RAH9-5676-5; Constanzo, 1828, en Cerrillo 2007; Guerra 1865, 31; CIL II, 835; García y Bellido, 1972-74, 63; idem, 1975, 234; CPILC, 377; Haba, 1986, n. ${ }^{\circ}$ 65; HEpOL, 21774; Esteban (CILCC III), 2013, n. ${ }^{\circ} 1004$.

—Línea 1: IVLIAE LVPERCI F. La I inicial se ha perdido; la V está prácticamente completa; a continuación hay un espacio donde parece observarse un fragmento central del asta vertical y otro del horizontal de la L, seguido de la I también completa; tras esta letra se aprecia el ángulo superior de la A y falta la E; hay un espacio de separación entre el gentilicio y la filiación, que ha perdido la L inicial; parece insinuarse la $\mathrm{V}$ subsiguiente con un color más oscuro y se ve parte inferior del asta recta de la P; la E solo conserva el trazo horizontal central y la parte inferior del asta vertical; de la $\mathrm{R}$ únicamente se aprecia también el extremo inferior del asta vertical y de la pata; la $\mathrm{C}$ y la I se han desprendido. Por último se observa claramente un amplio trazo algo inclinado, correspondiente al asta principal de la $\mathrm{F}$ final.

-Línea 2: LVPERCAE M FIDIVS. Aunque el grabado de la L inicial se ha perdido, aún se aprecian sus trazos en una tonalidad más oscura. La V y la P abierta se han conservado íntegramente; no así la $\mathrm{E}$, que solamente mantiene el asta vertical; tras ella se ve un pequeño trazo de la parte inferior del asta recta de la $\mathrm{R}$; la $\mathrm{C}$ y la $\mathrm{A}$ se insinúan en una coloración más oscura y la E final del cognomen conserva el asta vertical con esa misma tonalidad; a continuación hay una $\mathrm{M}$ - abreviatura del praenomen-, una $\mathrm{F}$ y una I casi completas seguida de un espacio en el que se ha desprendido el granito, tras el cual se ve nítidamente la $S$ final, que está precedida del trazo superior del asta de la $\mathrm{V}$.

2 En el texto de la inscripción las letras parcialmente visibles van con punto en la parte inferior. En la figura enmarcada se reconstruye el texto de la inscripción. En negro las letras o trazos de letras visibles en la actuali- dad, en distintas tonalidades de gris las letras o trazos de letras en forma de manchas oscuras dejadas por las filtraciones del agua, y en blanco las partes del texto que se han perdido. 
-Línea 3: MACER VXORI P I. La M inicial conserva perfectamente el trazo final y se insinúan los dos anteriores; de la A sólo se aprecia el ángulo de unión de las astas; a continuación se intuyen las letras $\mathrm{C}, \mathrm{E}$ y $\mathrm{R}$ por el cambio de tonalidad y más claramente la VX. Ni la I final de uxsori ni las abreviaturas P e I de la fórmula final se han conservado.

Las lecturas ofrecidas por los distintos autores que se interesaron por la inscripción, en general, no difieren mucho del original. Las variantes del texto parecen estar motivadas por el deterioro del soporte, aunque también hay que tener en cuenta el hecho de que algunos de estos autores nunca llegaron a ver la piedra y se limitaron a copiar la información ofrecida por terceros. Todo ello facilitó la cadena de errores en su interpretación y en la desubicación de la misma.

Llaman poderosamente la atención las dos letras finales de la última línea que solo viera Accursio entre los autores del siglo Xvi. Pudiera pensarse en un posible deterioro en su traslado a la casa del Deán en Plasencia que hiciera desaparecer estas grafías, pero el hecho de que reaparezcan en el manuscrito de Morales dos siglos después parece desechar tal posibilidad. El texto transmitido por Accursio reza así:

\section{Iuliae $\cdot$ Luperci $\cdot f / L$ upercae $\cdot M \cdot$ Fidius/Macer $\cdot$ uxori $\cdot p \cdot i$}

La lectura que hace Gaspar de Castro difiere poco de la de Accursio y, aparte de la omisión de las dos letras finales de la tercera línea, solo se diferencia en la inclusión de la $S$ de uxsori, el signo de puntuación tras la F final de la primera línea y la ausencia del mismo tras Macer y uxsori.

\section{Iuliae $\cdot$ Luperci $\cdot f \cdot /$ Lupercae $\cdot M \cdot$ Fidius/Macer uxsori}

Luis de Toro incluye una particular división de líneas, añadiendo una cuarta que ningún otro autor repite. No repara en la $S$ de uxsori ni en los signos de puntuación, que omite tras Iuliae en la primera línea y Macer en la tercera. Si puntúa, como Castro, tras la F de la línea 1.

\section{Iuliae Luperci $\cdot \mathrm{flLupercae} \cdot M \cdot$ Fi/dius Macer/uxori .}

Grutero no vio la inscripción y dice tomarla de un manuscrito enviado por Carlos (Gaspar) de Castro a Gutenstenio. Y efectivamente la versión que da es la de Castro, con la inclusión de la $S$ de uxsori, pero con la ausencia de la puntuación tras la $\mathrm{F}$ de la primera línea.

\section{Iuliae $\cdot$ Luperci $\cdot f / L$ upercae $\cdot M \cdot$ Fidius/Macer $\cdot$ uxsori}

Muratori dice copiar el texto de las schedae del P. Cattaneo, que no sabemos de dónde saca su versión corrupta del mismo y su procedencia de Brozas, lo que llevó a Mayans a considerarla una falsificación de Jerónimo Román de la Higuera. Cataneo o su fuente se inventa un supuesto Medius Fidius Macer que dedica la inscripción a su esposa Iulia Luperca y como la filiación no le cuadra la omite sin ningún escrúpulo. Faltan también en esta versión los signos de puntuación.

\section{Iuliae Lupercae/Medius Fidius/Macer uxori}


Mayans, al considerarla falsa, se limita a copiar el texto que Muratori incluye en su Thesaurus. Solamente difiere en el signo de puntuación final de la última línea que, más que parte del texto, parece el punto final del renglón con el que termina todas sus inscripciones.

\section{Iuliae Lupercae/Medius Fidius/Macer uxori.}

Ascensio de Morales ofrece prácticamente la misma versión que la de Accursio, con la fórmula final abreviada y se incluye la $S$ de uxsori que este no vio. Se omite la puntuación detrás de Iuliae y se coloca tras la F de la filiación y la I final.

$$
\text { Iuliae Luperci } \cdot f \cdot / \text { Lupercae } \cdot M \cdot \text { Fidius } / \text { Macer } \cdot \text { uxsori } \cdot p \cdot i \text {. }
$$

El texto que Constanzo toma del manuscrito de Boxoyo corresponde a la versión corrupta de la inscripción que circulaba en el siglo XviII que Muratori copia de Cataneo. Constanzo se limita a incluir los signos de puntuación detrás de cada palabra.

Iulia $\cdot$ Lupercae $\cdot /$ Medius $\cdot$ Fidius $\cdot /$ Macer $\cdot$ uxori .

Hübner da la misma lectura que Accursio. Como el italiano omite también la $S$ de uxsori:

Iuliae $\cdot$ Luperci $\cdot f /$ Lupercae $\cdot M \cdot$ Fidius/Macer $\cdot$ uxori $\cdot p \cdot i$

\section{El Pedestal izQUierdo del ARCo}

Antes de analizar el encaje del bloque de nuestra inscripción en el pedestal que sustentaba la estatua de Luperca, conviene hacer una serie de consideraciones acerca de su disposición en el conjunto arquitectónico ${ }^{3}$.

La estructura del pedestal izquierdo del arco es muy irregular y el planteamiento técnico no parece estar bien resuelto. Se conservan cuatro hiladas de sillares de las seis que debió de tener en origen, dispuestos a soga y tizón de una forma un tanto particular. La longitud de las hiladas es de $132 \mathrm{~cm}$ aproximadamente, pues el desplazamiento de los bloques impide tomar las medidas exactas. El lateral exterior se refuerza con una pilastra formada por bloques angulares que se prolongan hacia el interior del pilar y encajan en el propio pedestal, dándole una mayor consistencia y firmeza (fig. 3).

La primera hilada se compone de dos sillares moldurados, la segunda tres, la tercera y la cuarta dos y la quinta estaría formada por un solo sillar correspondiente al de la inscripción. Encima de este iba la sexta hilada correspondiente al coronamiento, con sillares también moldurados como el que se conserva en el lado derecho.

La profundidad de los sillares difiere sensiblemente unos de otros. Los dos primeros del pedestal van enjarjados en el pilar y alcanzan los $68 \mathrm{~cm}$ de profundidad; el tercero, de menores dimensio-

\footnotetext{
3 Un estudio completo sobre la visión histórica y gráfica del arco con toda la bibliografía sobre el monumento puede verse en: Cerrillo 2006, 305-316; las primeras excavaciones arqueológicas en el arco en: Bláz-
}

quez 1965, 23 ss.; un análisis más detallado sobre el arco en: García y Bellido 1972-1974, 45-90. Para las cuestiones arquitectónicas del el arco véase: NünnerichAsmus 1996. 


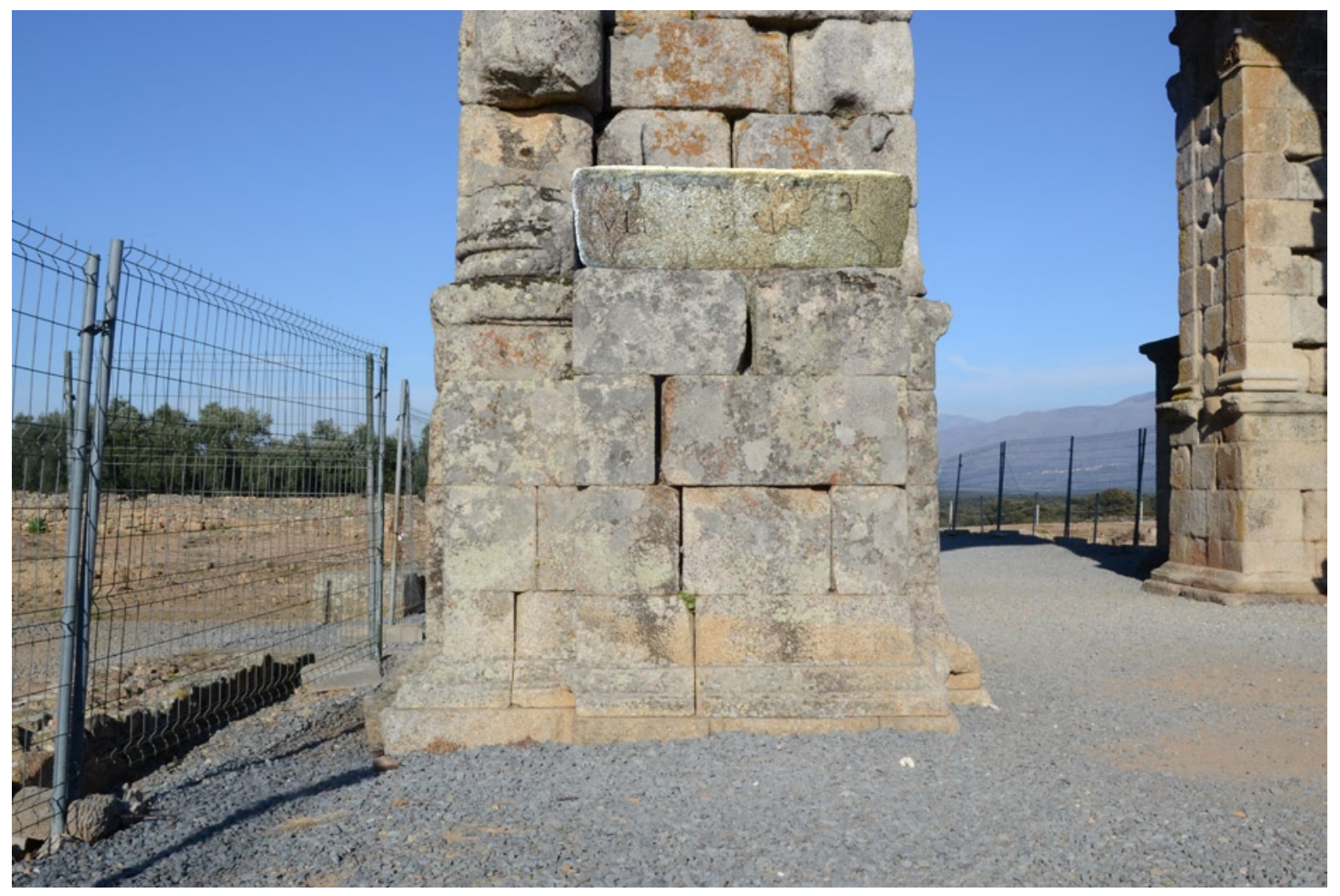

Figura 3. Reconstrucción del pilar izquierdo del arco

nes mide $56 \mathrm{~cm}$ y no llega a la vertical del pilar, que sobresale hasta emparejar con el del pedestal; y el cuarto mide unos $88 \mathrm{~cm}$ y va también enjarjado en el pilar. Sobre él iría el de la inscripción, de $33 \mathrm{~cm}$ de profundidad, que encaja en la basa de la semicolumna existente en el lateral izquierdo del pedestal; y encima el sillar o sillares del coronamiento moldurado, que muy bien pudieron estar dispuestos en forma de cuña a tenor de la escasa profundidad que tiene el bloque de la inscripción. Tampoco hay que descartar una quinta hilada formada por dos sillares de similar profundidad dispuestos a soga sobre los que iría la sexta hilada con otros a tizón.

Si observamos el estado de conservación del pedestal del lado izquierdo y lo comparamos con el del lado derecho, se percibe en aquel un importante desplazamiento de los sillares, sobre todo los de las dos hiladas superiores y, en menor medida, los de la segunda hilada que descansan sobre los bloques moldurados de la base. Todo parece indicar que las circunstancias de su estado de conservación se deben al robo de piedras en siglos sucesivos para su reutilización como material de construcciones. Desde luego, las dos hiladas superiores, que debían de estar sueltas, fueron arrancadas sin problemas de su lugar originario, pero las restantes permanecieron allí al estar unidas al pilar del arco. Seguramente los amplios intersticios que se aprecian actualmente se deban al intento de extracción de los sillares por parte de los amigos de lo ajeno. El pedestal derecho, sin embargo, se ha conservado prácticamente intacto al estar los sillares superiores enjarjados en el pilar (fig. 4). 


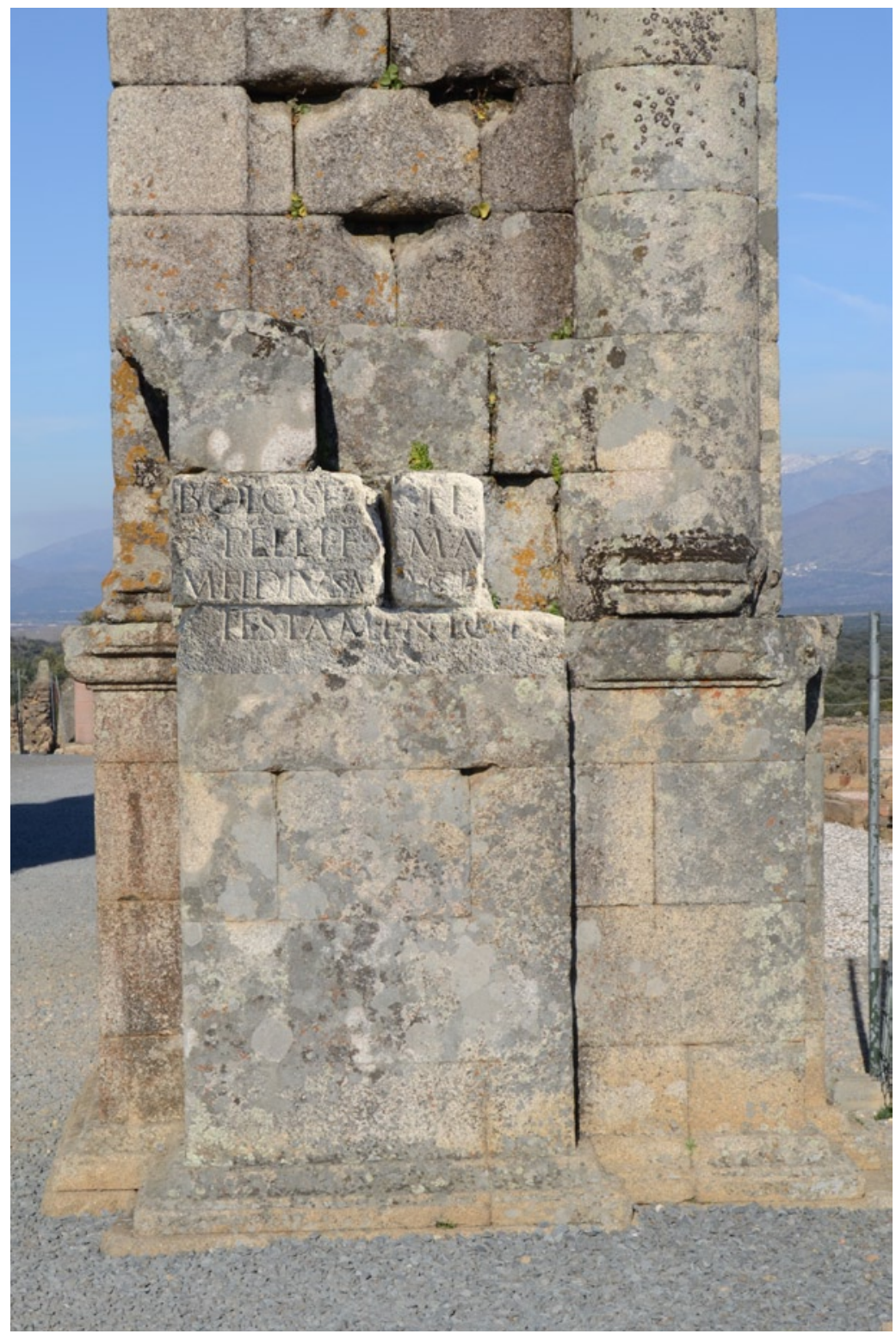

Figura 4. Pilar derecho con reconstrucción de la inscripción

Así pues, tanto la longitud como el grosor del sillar de la inscripción de Luperca encaja perfectamente en el conjunto del pedestal izquierdo; la diferencia mínima de $1 \mathrm{~cm}$ puede explicarse, como hemos señalado, por el desplazamiento de los sillares que es a todas luces evidente. Igualmente, todos los sillares, incluido el de la inscripción, miden $43 \mathrm{~cm}$ de grosor y el hecho de que su profundidad de $33 \mathrm{~cm}$ sea menor que la del resto no es significativo, pues cada uno de ellos es diferente a los demás. El espacio que actualmente existe en la poyata de la cuarta hilada es de $60 \mathrm{~cm}$, por lo que caben dos posibilidades: o bien tras el correspondiente a la inscripción había otro sillar, o qui- 
zás era un mismo bloque que después de su extracción fue convenientemente retallado para su transporte y reaprovechamiento en los muros de la casa del Deán.

\section{M. FidiUs MACER y IULIA LUPERCA, REPRESENTANTES DE LA ELITE LOCAL DE CAPERA}

No viene al caso hacer aquí un estudio exhaustivo de la inscripción y de la personalidad del duunviro, por cuanto otros investigadores se han ocupado suficientemente del tema ${ }^{4}$. Sí me parece conveniente ofrecer unas breves pinceladas sobre las familias de ambos personajes y el significado del monumento.

Como hemos podido demostrar con este trabajo, la inscripción que nos ocupa formó parte del pilar izquierdo del arco y rotulaba con toda probabilidad el pedestal de la estatua de Iulia Luperca, esposa del primer magistrado de la ciudad que en la inscripción que aún se conserva en el pilar derecho dedica una escultura similar a sus progenitores. Las excavaciones arqueológicas realizadas en los pilares del lado opuesto descubrieron los cimientos de los pedestales de mayor tamaño (Blázquez 1965, 25), donde debían de descansar sendas estatuas ecuestres, quizás del propio duunviro.

Se puede considera el arco — el único conocido en Hispania con financiación privada- como un monumento a la memoria de una de las familias más relevantes de la ciudad: los Fidii Macri. La inscripción del pilar derecho especifica su construcción ex testamento, no sabemos a ciencia cierta si por voluntad expresa de los padres del duunviro o por cumplimiento en vida de una de las disposiciones testamentarias del propio magistrado ${ }^{5}$. Con este acto de munificencia pretendía no tanto su promoción política, que ya estaba asegurada, cuanto reafirmar su estatus social y el de su familia al frente de la comunidad, al mismo tiempo que celebrar el prestigio de su estirpe y la fama y honores alcanzados por uno de sus miembros más ilustre. Su majestuoso alzado a la entrada del foro presidiría en el futuro la vida municipal y su disposición sobre la calzada suponía un acto de propaganda a la vista de todos los viajeros que transitaran por esta importante vía de comunicación.

Macer descendía de una familia local romanizada ya al menos desde dos generaciones atrás, pues su abuelo paterno — Macer — y su padre — Fidius — habían adoptado nombres romanos. Una familia que había contribuido decisivamente a la consolidación urbana de Capera, que había participado en la administración local bajo modelos romanos cuando no era más que un oppidum estipendiario y que abogaría en favor de la concesión del estatus de municipio de derecho latino en época de los flavios. Macer es un cognomen romano muy extendido en Hispania y cuenta con un buen número de testimonios en Lusitania (Navarro-Ramírez 2003, 221). El cognomen Fidius debe de ser una adaptación del romano Fidus, pues no se repite, que sepamos, en la epigrafía peninsular (Abascal 1994, 138).

4 El trabajo más completo y reciente sobre la personalidad de $M$. Fidius Macer y la epigrafía con él relacionada es el de González Herrero (2002, 417-433; 2004, 365-384).

5 Bolosea[e] Fi[dio] Pelli.f(iliae). Ma[crif(ilio)] M(arcus) $\cdot$ Fidius $\cdot$ Mace $[r]$ [(?) ex] testamento $f$ (aciendum) $c$ (uravit)
Véase CILCC III 1003 (CIL II 834; $A E$ 1967, 197; CPILC 183; HAE 2574; HEp 12, 93).

Étienne y Mayet (1971, 388-389) dan otra versión de la inscripción: Bolose[e] Fi[di]/ Pelli f(iliae) Ma[cri n(epoti)] M(arcus) Fidius Macer/testamento p[oni] i(ussit), en la que habría una única destinataria, Bolosea, hija de Fidius Pellius y nieta de Macer. 
Como indica la inscripción del templo de Trebaruna $^{6}$, nuestro personaje había desempeñando sus funciones de magistratus de la primitiva comunidad estipendiaria cuando la ciudad fue beneficiada con el cambio de estatus, siendo honrado por dos veces con el duunvirato, suprema magistratura municipal del nuevo municipio latino. Su posición económica y su nacimiento le habían permitido formar parte del ordo local y desempeñar las funciones más altas en la administración de la ciudad, lo que le abrió las puertas de la ciudadanía romana per honorem e incluso su promoción al orden ecuestre, como así lo pone de manifiesto el título de praefectus fabrum, reservado a la clase de los caballeros?.

Nada sabemos de sus ascendientes por vía materna, solo que mantenían sus tradiciones indígenas, al menos en lo que a la onomástica se refiere. Estaban romanizados, pero mantienen cierta reticencia a las nuevas modas que hacía tiempo había asumido ya la rama paterna. Hemos de suponer que el matrimonio de Fidius y Bolosea (padres de Macer) se había llevado a cabo de común acuerdo entre familias de la elite local de rango similar y que esta unión vendría a potenciar el peso específico de ambas familias en el seno de la aristocracia local. El indigenismo de ambos progenitores parece evidente, pues llevan nombres que, hasta el momento solo se han documentado en Lusitania. Del cognomen Fidius ya hemos hecho referencia en líneas anteriores y sobre Bolosea, con esta variante, solo se tienen dos testimonios más procedentes de San Martín del Castañar-Salamanca (CIL II 881) y Vila Fernando-Guarda (Osório 2006, 365) ${ }^{8}$.

Tras alcanzar la ciudadanía romana después de desempeñar la suprema magistratura ya en el nuevo municipio latino, nuestro personaje adaptó su estructura onomástica peregrina al de un auténtico ciudadano de Roma. Eligió el praenomen Marcus, convirtió el nombre de su padre en su nomen gentile y el de su abuelo en su cognomen y fue inscrito en la tribu Quirina. Tradicionalmente los beneficiados con la condición de civis adoptaban el gentilicio del emperador que les había promocionado, aunque en este caso Macer decidió honrar a sus antepasados recurriendo a sus nombres.

Nuestro duunviro se había unido a Iulia Luperca que, hemos de suponer, era también miembro de una familia de la elite local. El gentilicio Iulius parece indicar una cierta vinculación de sus ascendientes con el proceso romanizador que supuso la urbanización del territorio situado al norte del Tajo en época de César y Augusto. Seguramente la familia de Luperca se había mostrado partidaria de los intereses romanos en la zona y fue recompensada con su aceptación en el seno de la aristocracia local. El nombre de su padre y el suyo propio, Lupercus/a, es romano, aunque la filiación cognominal indica que no era ciudadana romana sino peregrina y que accedió a su nuevo estatus tras la promoción jurídica de su esposo ya en época flavia. Este cognomen, aunque no es muy frecuente en la epigrafía hispana, se repite en tres inscripciones más procedentes de Lusitania ${ }^{9}$ y en otras tantas de la Citerior ${ }^{10}$.

6 Aug(ustae). Trebar[une]/M(arcus). Fidius. Fidi. f(ilius). Quir(ina tribu) [Macer]/mag(istratus). III. IIvir. II. praef(ectus) fa[brum], en CILCC III 1014 $($ CPILC 818; $A E$ 1967, $197=A E$ 1987, 616; HAE 2574; $A E$ 2002, 705; HEp 12, 93).

7 Sobre la pertenencia de los praefecti fabrum al orden ecuestre véase el trabajo reciente de Âlvarez Melero $2013,1-32$, en el que se ofrece una detallada bibliografía al respecto.

${ }^{8}$ La forma Bolosa está documentada en inscripciones de Coria-Cáceres (CILCC IV, 1189), Villar del ReyBadajoz ( $A E$ 1971, 146b) e Idanha-a-Velha-Castelo
Branco (CIL II, 440); algunos casos incompletos son los de Tomar-Santarem (AE 1998, 699), Condeixa-a Velha-Coimbra (Etienne-Fabre-Lévêque-Lévêque 1976, 329). Un Bolosi se documenta también en Idanha-a-Velha (HAE 1108). Consideraciones de carácter lingüístico de este antropónimo se pueden seguir en Vallejo 2005, 213-214.

9 Mérida (IHC 21), Talavera de la Reina (CIL II 917) y Hinojosa del Duero-Salamanca (HAE 1280).

10 Polán-Toledo (HEp 7, 1997, 976), Prat de ReyBarcelona (CIL II 4479) Tarragona (CIL II 4104). 


\section{Cuestiones historiográficas}

Desde que Mariangelo Accursio, llegado a la corte de Carlos V entre 1525-1526, copiara la inscripción de Iulia Luperca, son muchos los autores que han tratado sobre ella, aunque no todos la vieron. El humanista italiano visitó la zona en 1527 y en un manuscrito que se conserva en la Biblioteca Ambrosiana de Milán, la ubica en Oliva de Plasencia, donde la vio en la pared de la casa de Pedro de la Cerda. Dice además que se encuentran desparramados numerosos fragmentos de epigramas y piedras llevadas todas de Cáparra a distancia como una legua a la izquierda, en la villa de Oliva ${ }^{11}$.

Se desconoce el momento exacto y las circunstancias del acarreo de piedras procedentes de las ruinas de Cáparra a la cercana villa de Oliva de Plasencia, pero de las palabras de Accursio parece desprenderse que las piezas movidas no eran piedras cualesquiera, sino fundamentalmente «epigramas» y los bloques mejor labrados.

Quizás el viaje de Accursio a Oliva en 1527 coincidiera con el momento del acarreo de las piedras para su posterior traslado, pero en cualquier caso no permanecieron mucho tiempo en el lugar, pues algunos años después, la inscripción que nos ocupa al menos, aparece en la casa del Deán en Plasencia.

Allí la vio Gaspar de Castro, beneficiado de la catedral salmantina y natural de Ledesma, en su viaje a Plasencia en 1550, sobre el que en una carta fechada el 26 de abril de 1551 informa a Antonio Agustín, Auditor de La Rota, dándole noticias de las inscripciones romanas de Extremadura ${ }^{12}$. Entre ellas se encontraba la de Luperca. El canónigo salmantino dice en su viaje a Plasencia:

Ningunas señales de antiguedad vi en este pueblo, y aunque copié en el estos nueve letreros dizenme que un Dean de aquella ciudad estudioso de cosas antiguas los truxo delas ruinas de Caparra y de aquel contorno, y los puso en su casa, donde yo los hallé parte en un juego de pelota y parte un una esquina dela casa. Encima del texto rotula: in lapide communi.

No es seguro que todas las inscripciones que se hallaban en la casa del Deán fueran acarreadas por este desde las ruinas de Cáparra como afirma Gaspar de Castro, pues la referencia parece un tanto imprecisa. Es muy posible que algunas de ellas fueran fruto de donaciones realizadas por algunos nobles placentinos en un momento en el que el trasiego de este tipo de objetos era frecuente.

Dos décadas después, Luis de Toro la incluye entre las inscripciones de Plasencia en un manuscrito fechado en 1573, conservado actualmente en la biblioteca de la Universidad de Salamanca ${ }^{13}$. Nacido en Plasencia, fue médico personal de Pedro Ponce de León, Inquisidor General y obispo de Plasencia y protegido de Luis de Zúniga, Marqués de Mirabel. El galeno y humanista placentino da una relación de las inscripciones existentes en Plasencia en aquella época. Primeramente se refiere a la colección del Marqués de Mirabel y seguidamente alude a las ocho que se encontraban

11 Accursio en el fol. 205r dice: "Año de 1550. Fui a la provincia de Estremadura, y estuve en Coria, Cáceres, Mérida, Plasencia, Caparra, y en estos lugares vi y copie las memorias antiguas, que van en este pliego como en las piedras se contiene».

12 Carta de Gaspar de Castro a Antonio Agustín fechada el 26 de abril de 1551, Ms. RAH 9-6002-9a, copia del siglo XVIII del original del Vat. Lat. 6040, 1503-212v. (cf. CIL II, p. IX, n. 12 . Mas.-12 p. en 4. ${ }^{\circ}$ fol. 291r.-296); en el folio 293v se cita la inscripción mencionada (el 207r. en el original). Sobre el epistolario de Antonio Agustín véase: Carbonell 1991, 75-92.

13 Ms. Placentiae urbis et eiusdem episcopatus, descripctio 1573, cf. Sayans 1961. También recoge este manuscrito Sánchez Loro 1982, 179, lápida II. 
en la casa del Deán, señalando que son piedras sacadas de Capera ${ }^{14}$. Aunque su condición de placentino le posibilitaría ver la inscripción, la versión que ofrece en cuatro líneas y el hecho que la describa como Castro: in lapide communi, me hace sospechar si realmente la tuvo delante o simplemente la copió del propio Castro. Sea como fuere, no debió de poner mucha atención a la hora de copiar el texto, pues no se adecua al original.

Grutero (1602 y 1793, 6), que a comienzos del siglo XviI editó su corpus de «Inscripciones Antiguas» a partir de fuentes manuscritas y de ediciones anteriores, incluye el epígrafe de Luperca, aunque no la vio. El corpus gruterino recoge las inscripciones citadas por Mamerano que fueron incorporadas después a las Iscriptiones veteres in Hispania repertae de Adolfo Occo (1596). Ni Mamerano ni Occo recogían entre sus inscripciones nuestra piedra, luego Grutero tuvo que utilizar otra fuente distinta a las anteriormente citadas. El epigrafista holandés señala en sus Inscripciones Antiquae publicada en 1602: Grutero ex Caroli de Castro autor. Gutenst.; es decir que tomó la información que Leonardo Gutenstenio le envió a partir de un manuscrito de Carlos de Castro (Mommsem 1878, 67-77). Es posible que se refiera a Gaspar de Castro, aunque es poco probable pues no la ubica en Plasencia — como dice el propio Castro- sino en la localidad de Galisteo, a pocos kilómetros de Cáparra, y no parece que la piedra haya estado allí en ningún momento, al menos no hay constancia de ello.

Se sabe de la afición de algunos nobles durante el siglo Xvi por las antigüedades romanas siguiendo la corriente humanista del Renacimiento. Significativa fue la actividad coleccionista de los Condes de Osorno en su Señorío de Galisteo o la de los Marqueses de Mirabel en Plasencia, que crearon en sus palacios auténticas colecciones de mármoles fruto del expolio de yacimientos cercanos (Tornay 2012-2013, 25-40; Edmondson 2001a, 107). Don García Fernández Manrique, III Conde de Osorno, llevó a Galisteo estatuas e inscripciones traídas desde Mérida, según cuenta Mamerano (Epitaphia, fol. [35]r) y no tenemos noticias de que entre ellas hubiera piezas procedentes de Cáparra, aunque lo más probable es que también llegaran algunas con este origen. También Don Pedro de Ávila y Zúníga, Marqués de Mirabel, era ávido coleccionista de antigüedades e hizo acopio de piezas procedentes de Mérida y quizás de Cáparra que aún se conservan en el Pensil de su palacio placentino ${ }^{15}$.

Si en 1527 Accursio la vio en Oliva y en 1550 Gaspar de Castro la sitúa en la casa del Deán, no se puede descartar que en el ínterin el III Conde de Osorno la acarreara al convento dominico de Galisteo. Sobre este convento y las andanzas del Conde nos informa Mamerano en sus Epitaphia et Antiquitates Romanorum per Hispaniam, obra que no llegó a editarse pero de la que se conservan dos versiones manuscritas ${ }^{16}$. Nik Wagener "Mameranus», historiador luxemburgués, cronista de Carlos V, viajó por tierras de Extremadura y recaló en Galisteo. Solo pudo describir un total de trece inscripciones que se hallaban en el palomar del citado convento, pues el encargado de vigilarlas no le permitió seguir con su estudio de los epígrafes allí depositados (Epitaphia, fol. [38]v).

14 Et quoniam de huiusmodi vetustatis monimentis fit mentio, no videbitur alienum aut praeter operis institutum, et alia addere, quea in Decanorum domo, illustri quidem maximorumque ingeniorum nenitrice, excussa lapidibus visuntur, ex Capera, mas. L. de Toro, fol. 21, cf. Sayans 1961, 52.

15 En el palacio del Marqués de Mirabel se conservan ocho inscripciones (CIL II, 78*, 501, 536, 562,
839; CILCC III, 1092, 1093 y 1099, de las cuales tres pueden proceder de Cáparra (CILCC III, 1092, 1093 y 1099). Sobre esta colección véase: Marcks-Jacobs, 2001, 155-208.

16 Manuscritos conservados en Ausburgo (Staats- und Stadtbibliothek 4. ${ }^{\circ}$ Cod. H(alder) 26) y en Hannover (Niedersachsische Landesbibliothek, ms. XXVIII 1653). Sobre Mameranus véase: Vannérus 1949, 299-321. 
No se conoce el año en que Mamerano anduvo por tierras cacereñas, pues los investigadores no se ponen de acuerdo, pero debió de tener lugar entre 1533 y 1535. Quizás la inscripción de Luperca estaba en el palomar entre las que no pudo transcribir el luxemburgués, pues no la menciona en sus Epitaphia.

Sabemos que por aquellas fechas algunas piezas en posesión del Conde de Osorno cambiaron de dueño, regaladas a miembros nobles de su círculo de amigos ${ }^{17}$. Este fue el caso de la inscripción del pedestal de estatua, también de Cáparra, que Cocceia Severa mandó poner en honor de su abuela Avita ${ }^{18}$.

Una de estas familias nobles era la de los Zúñiga, Condes de Plasencia y Marqueses de Mirabel. Don Fadrique de Zúniga y Sotomayor, I Marqués de Mirabel, tenía bajo su protección a la familia de Don Diego de Jerez, deán de la catedral de Plasencia durante 1482-1505 (González 1932, 87). Uno de sus hijos con la unión de María Monreal fue Don Gómez de Jerez, a la sazón también deán de la catedral, al igual que el hijo de este, Don Alonso de Jerez. Aunque no se puede descartar que fuera Don Gómez, deán entre 1525-1526, el autor del traslado, lo más probable es que fuera Don Alonso, deán entre 1527-1550, quien trajera a su casa de Plasencia las inscripciones que Accursio había visto en Oliva y al que se refiere Gaspar de Castro en su carta.

Salvo la obra de Grutero, no tenemos ninguna otra referencia de la inscripción en el siglo XvII, aunque desde finales del siglo anterior proliferan los "falsos cronicones», que apoyándose en datos espurios, pretenden reinterpretar la Historia. Es el momento de la aparición de textos epigráficos inventados y la reubicación de inscripciones auténticas con el fin de engrandecer los orígenes ciertas localidades. Estos falsos cronicones confundieron a no pocos de los autores posteriores que recurrieron a estas fuentes para completar los corpora epigráficos que empiezan a proliferar en los siglos XVIII y XIX. De este modo, la inscripción de la caperense Luperca sufrió por mor de estas crónicas un peregrinaje de ubicaciones seculares por distintas poblaciones cacereñas.

Así, a comienzos del siglo XVIII Luigi Antonio Muratori la ubica erróneamente en Brozas: in oppido les Brocas, agri Alcantarae. El jesuita italiano tampoco vio la inscripción, pues en su Thesaurus dice tomarla $e$ schedis P. Cattanei (Muratori 1740, 1356, 5) ${ }^{19}$.

Mayans en su biografía de El Brocense (Mayans 1746, 4) la incluye entre las inscripciones falsas inventadas por Jerónimo Román de la Higuera a finales del siglo xvi, quien la ubica en Brozas ${ }^{20}$. Que el clérigo era un falsario no hay duda ninguna y está suficientemente demostrado (Caro Baroja 1991, 161-178), pero no todas las inscripciones que menciona eran fruto de la mente manipuladora del clérigo. No lo era, desde luego, la inscripción de Luperca y, probablemente, tampoco alguna más de las que aparecen junto a ella.

\footnotetext{
17 Una de estas piezas lleva una inscripción al dorso donde se especifica la donación del IV Conde de Osorno, D. Pedro Manrique. Este es el caso del monumento funerario de Iulianus, que en su reverso puede leerse: «Esta antigualla me dio el mui illustre señor Don Pedro Manrique, conde de Osorno, 1558. Hallóse en Mérida 1530» (CIL II 562). Sobre el particular véase: Edmondson 2001, 139-141.

18 CIL II $813=$ CPILC $186=$ CILCC III 1005. El pedestal de mármol terminó en el castillo que D. Pedro de Ávila y Zúniga, hermano del Marqués de Mirabel, tenía en la localidad abulense de las Navas del Marqués. Sabemos también que D. Pedro de Ávila y Zúñiga tenía
}

una gran amistad con D. Pedro Fernández Manrique y Cabrera, IV Conde de Osorno y Duque de Galisteo, que muy bien pudo ser el poseedor de la piedra y quien la regalara a este. La mencionada inscripción se encuentra en el Museo Arqueológico Nacional desde 1914, donde se expone en una de sus salas (n. ${ }^{\circ}$ de registro 20222).

19 El padre Cayetano Cattaneo era otro jesuita natural de la ciudad italiana de Mesina que vivió entre 1695-1733 y participó activamente en las misiones de América del Sur.

20 Jesuita nacido en Toledo en 1538, Doctor en Teología y autor de Cronicones que abarcan desde la Antigüedad hasta la época altomedieval. 
En 1743 Felipe V comisionó a Ascensio de Morales y Tercero para investigar el archivo municipal y el catedralicio, recabando la información sobre monumentos de Plasencia (Rodríguez 1931). En su estancia en la ciudad copió los textos de las inscripciones existentes en el palacio del Marqués de Mirabel y en la casa de los Vargas, los cuales se incorporaron a un manuscrito que se conserva actualmente en la Real Academia de la Historia (Morales 1753, RAH 9-5676-5). Morales es el único autor que da detalles de su forma y de su ubicación en el edificio. En su manuscrito dibuja un bloque rectangular (n.o 14) y dice: Piedras de grano de la misma figura que aqui tienen: y están con las tres siguientes en la esquina dtras casas de Don Antonio de Vargas, que mira al Poniente y Medio dia.

En 1771 el padre Enrique Flórez visitó Plasencia y en sus escritos hace referencia a las inscripciones que allí vio, aunque no da sus textos, como es frecuente en otros muchos lugares a los que viajó, y dice: Al redor de la casa de Don Luis de Bargas están colocadas once Inscripciones Romanas, y en casa del Señor Marques de Miravél hay hasta siete, que son sepulcrales: y diversas figuras del tiempo de los Romanos (Méndez 1771, 264) ${ }^{21}$.

A comienzos del siglo xix Claudio Constanzo reproduce un manuscrito elaborado por SimónBenito Boxoyo sobre la epigrafía de Cáceres, terminado el 1 de junio de 1800. La minuciosidad con que Boxoyo describe las inscripciones que tiene delante contrasta, como señala Cerrillo ${ }^{22}$, con la parquedad con que trata las enviadas por sus informadores, de tal manera que es fácil identificar cuáles fueron copiadas por mano propia. Desde luego no pudo ver nuestra inscripción, porque nunca estuvo en Brozas. Sabemos que Boxoyo, en su afán por reunir el mayor número de inscripciones, recurrió a otros manuscritos e informadores de dudosa fiabilidad que le llevaron a cometer errores en la ubicación de algunas de ellas. Este es el caso de varias inscripciones que estaban en Plasencia procedentes de Cáparra, entre las que se encuentra la de Luperca, que equivocadamente la sitúa también en Brozas junto con la de Cocceia Severa.

Posiblemente la referencia de la ubicación en Brozas de las inscripciones que Cocceia Severa dedica a su abuela Avita y a su madre Trebia Procula, se deba al "pseudo Fernández Franco», un falsificador y seguidor de la línea de Román de la Higuera, que a mediados del siglo XviII intentó dar carta de naturaleza a sus inscripciones falsas inventándose una serie de cartas que atribuye al bachiller Juan Alonso Franco o Juan Fernández Franco — con estos dos nombres aparece su obra manuscrita-, discípulo del historiador y cronista del siglo XVI, Ambrosio de Morales (Gimeno 1997). Cerrillo cree, por su parte, que las inscripciones supuestamente procedentes de Brozas están tomadas de la obra de Torres Tapia (Cerrillo 2007, 77).

En 1854 se edita la primera edición de Notas a las Antigüedades de Estremadura de D. José de Viu, de Felipe León Guerra. Viu no incluye la inscripción en su repertorio, pero Guerra introduce un comentario en el que cita a Franco, del que dice aludir a la inscripción de Luperca ${ }^{23}$. Sin duda ninguna se refiere al "pseudo Fernández Franco» anteriormente citado, pues ofrece la misma lectura y la ubica también en la localidad de Brozas. Guerra, desde luego, no vio el manuscrito de Constanzo porque en su comentario sobre la misma dice: y creo que también la pone Constanzo (Guerra 1865, 31).

21 Este sacerdote inició su viaje desde Madrid, en compañía de fray José Ávila, el jueves 23 de mayo y llegó a Plasencia el último día del mes o primeros de junio, permaneciendo en la ciudad hasta el 7 de junio, cuando regresó a la capital (p. 264).
22 Un minucioso estudio sobre el manuscrito de Boxoyo y el papel de Claudio Constanzo en su transmisión puede encontrarse en Cerrillo 2007.

23 Franco pone igualmente en Brozas una que dice: Juliae Lupercae Medius Macer uxori, cfr. Guerra 1854, 36. 
Hübner visitó tierras extremeñas y recaló en Plasencia en su primer viaje a España realizado entre 1860-1861. Allí tuvo oportunidad de ver la colección de inscripciones del palacio de Mirabel y las conservadas en la casa del Deán, aunque me asaltan serias dudas de que reparara en la de $L u$ perca, pues al referirse en el n. ${ }^{\circ} 835$ del CIL al lugar de ubicación de nuestra inscripción en la casa del Deán utiliza una lacónica expresión: ibi extat. También dice descripsi, pero resulta cuando menos sorprendente que no especificara el lugar exacto del edificio en que se encontraba y, lo que es más extraño, que un epigrafista avezado como él no la leyera correctamente. Desde luego no vio la $S$ de uxsori que leyeron Gaspar de Castro y Ascensio de Morales. Descartando un error del alemán, solo se me ocurre una excusa para esta omisión: que el texto estuviera lo suficientemente deteriorado como para impedir una buena lectura y lo hiciera a partir de la versión de Accursio.

Esta es la información que poseemos de nuestra inscripción hasta la elaboración del CIL a mediados del siglo XIX. Recapitulando puedo decir sin temor a equivocarme que desde el siglo XVI, con la excepción de Ascensio de Morales y la un tanto dudosa de Hübner, ningún viajero que visitó Plasencia en los siglos posteriores consiguió ver la piedra. Ninguna referencia hay sobre ella entre los autores del siglo XVII. Tampoco los ilustrados del xviII que se acercaron a la localidad hablan de ella. No lo hizo Ponz, viajero incansable que en 1771 inicio su viaje por España y en su paso por tierras extremeńas recaló en Plasencia. Allí da los textos de cinco inscripciones que había en la casa de los Vargas, entre los que no estaba el de Luperca (Ponz 1784, 124-125) ${ }^{24}$.

Tampoco los autores del XIx, salvo posiblemente Hübner, acertaron a verla. No lo hace CeánBermúdez que en 1832 publica el Sumario de Antigüedades en el que se mencionan algunas inscripciones placentinas. En él se dice: El caballero Vargas reunió en su casa inscripciones romanas miliarias, de dedicaciones, y sepulcrales, de las cuales solamente copiaré tres (Ceán-Bermúdez 1832, 417). Entre ellas no está la de Luperca. Ni Viu, que a mediados de la centuria edita su obra, que recoge un importante número de inscripciones extremeñas, entre ellas las que había en la casa de los Vargas (Viu 1846 y 1842).

Y desde luego no hay noticias de que alguno de los muchos eruditos e investigadores del siglo xx interesados en la epigrafía placentina hayan manifestado tenerla delante. El catálogo de Mélida no la incluye (1924). En la década de los 60 se editan los tres tomos de Blázquez sobre las excavaciones de Cáparra y en el segundo incluye algunas inscripciones procedentes del yacimiento localizadas en los municipios próximos, pero no habla de ella (1966). García y Bellido, en su estudio sobre el tetrapylon de Cáparra, analiza la inscripción y se limita a citar la referencia de Accursio (1972-1974, 45-90). El placentino Sánchez Paredes (1976, 85-98), que recoge inscripciones romanas de la zona, tampoco lo hace. $\mathrm{Ni}$ el prolijo don Carlos Callejo ${ }^{25}$, que estudió más de un centenar de inscripciones de la provincia de Cáceres, consiguió dar con el paradero de nuestra inscripción. El corpus de Hurtado de San Antonio la incluye, pero el autor no la vio, limitándose a citar obras anteriores (Hurtado 1977, n.o 377). En la década de los 80, Haba realiza su tesina de licenciatura sobre el partido judicial de Plasencia e igualmente la incorpora, pero la da como desaparecida (1986, n. ${ }^{\circ}$ 65).

Cuáles puedan ser las causas de que muchos de los autores que visitaron la ciudad de Plasencia y se interesaron por la epigrafía del lugar no vieron la inscripción de Luperca, se nos escapan, pero, ante la visión del propio bloque que contiene el texto, se nos plantean dos posibilidades que

\footnotetext{
24 Dice Ponz: En la casa que llaman de los Vargas, hay otra porción de inscripciones romanas, que probablemente se traxeron de Caparra... y da los textos de cinco. os de cuatro, todas ella proceden de la Casa del Dey otra porcil.
}

25 Callejo 1962, 111-132; id. 1965, 11-82; id. 1967, 85-121; id. 1970, pp. 132-168; entre las más interesantes. 
no son excluyentes. En primer lugar el deterioro de la capa superficial del granito, que debió de estar en fase muy avanzada ya en el momento en que los estudiosos arriba mencionados se acercaron a los muros de la Casa del Deán. Este mismo deterioro se puede apreciar hoy día en mucho de los sillares de la base del arco, que aparecen exfoliados y con la capa exterior desprendida. Y, en segundo lugar, es muy posible que en las sucesivas remodelaciones realizadas en el edificio la inscripción fuera total o parcialmente encalada, como así parece desprenderse de la capa blanquecina que aún se conserva en los bordes del sillar. Cualquier intento de limpieza posterior habría contribuido a acelerar el proceso de deterioro.

En los numerosos viajes que he hecho a la capital del Jerte he tenido la ocasión de hablar con algunos de los sacerdotes más ancianos que residen en la Casa Sacerdotal (antigua Casa del Deán). Pero ninguno ha sabido darme noticias de la ubicación de la inscripción; solo alguno dice conservar vagos recuerdos de unas piedras inscritas que estaban en sus muros. Este es el caso D. José Sendín, fallecido mientras elaborábamos este trabajo, que aún mantenía en la memoria la posición que ocupaban algunas de ellas, aunque no pudo identificarlas ${ }^{26}$.

\section{BiBLIOGRAFÍA}

Abascal Palazón, J.M., 1994, Los nombres personales en las inscripciones latinas de Hispania, Madrid.

Accursio, M., 1550, Itinerarium ab Olmedo (prope Medina del Campo) ad divam Guadalupiam et inde ad Emeritam et pleraque alia loca, ms. O-125, Biblioteca Ambrosiana, Milano.

Álvarez Melero, A., 2013, «Los praefecti fabrum oriundos de las provincias hispanas. Una nueva aproximación», Espacio, Tiempo y Forma, Historia Antigua, 26, 123-154.

Blázquez Martínez, J.M.a , 1965, Caparra I, [Excavaciones Arqueológicas en España], Madrid.

—, 1966, Caparra II, [Excavaciones Arqueológicas en España], Madrid.

Callejo Serrano, C., 1962: «Novedades y correcciones a la epigrafía de Cáceres», BRAH 161, 111-132.

—, 1965, «Aportación a la epigrafía del campo norbense», BRAH 157, 11-82.

—, 1967, "Cédulas epigráficas del campo norbense», Zephyrus 18, 85-121.

—, 1970, «Nuevo repertorio epigráfico de la provincia de Cáceres», AEspA 43, 132-168.

Carbonell i Manils, J., 1991, Epigrafia i numismàtica a l'epistolari d'Antonio Agustín (1551-1563), 2 vols. Tesis doctoral dirigida por Dr. Marc Mayer i Olivé, Universidad Autónoma de Barcelona, Barcelona.

Caro Baroja, J., 1991, Las falsificaciones de la Historia (en relación con la España), Barcelona.

CeÁn-Bermúdez, J.A., 1832, Sumario de las Antigüedades Romanas que hay en España, en especial las pertenecientes a las bellas Artes, Imprenta de D. Miguel de Burgos, Madrid.

Cerrillo Martín de CáCeres, E., 2006, «El tetrapylon de Cáparra. Visión histórica y gráfica», Zephyrus 59, 305-316.

2007, Claudio Constanzo y la epigrafía extremeña del siglo XIX, Madrid.

Constanzo, C., 1828, Colección de Lapidas y otros Monumentos Antiguos descubiertos y averiguados en esta Villa de Cáceres, sus Ynmediaciones, y en otras Poblaciones de la Provincia de Estremadura hasta hoy Primero de Junio de este año de Mil Ochocientos Por D. Claudio Constanzo Socio de la Academia de la Historia 1828, Cáceres.

Edmondson, J., 2001a, «El redescubrimiento de los monumentos funerarios con retrato. Siglos XVI-XX», en Imagen y Memoria. Monumentos funerarios con retrato en la Colonia Augusta Emerita, Madrid.

26 Estaremos siempre agradecido a D. José Sendín Blázquez por la amabilidad con que nos acogió en nuestra visita a Plasencia, así como sus sabias informaciones sobre la historia reciente de la antigua Casa del Deán. Descanse en paz. 
—, 2001b, «Catalogue of funerary monumentes with portraits», en Imagen y Memoria. Monumentos funerarios con retrato en la Colonia Augusta Emerita, Madrid, 113-187.

Esteban Ortega, J., 2013, Corpus de inscripciones latinas de Cáceres III. Capera [CILCC III], Cáceres.

Étienne, R., G. Fabre, M. LÉvêque, P. LÉvêque, 1976, Fouilles de Conimbriga II. Épigraphie et sculpture, Paris.

Étienne R., F. Mayet, 1971, «Du nouveau sur Capera-Capara», REA 73 (3-4), 382-290.

Floriano Cumbreño, A., 1944, «Excavaciones en la antigua Caparra (Cáparra, Cáceres)», $A E s p A$ XVII, n. ${ }^{\circ} 56,270-286$.

García Vidal, C., 1946, El seminario de Plasencia. Apuntes para su historia, Plasencia.

García y Bellido, A., 1972-1974, «El tetrapylon de Capera (Cáparra, Cáceres», $A E s p A$ 45-47, 45-90.

Castro, G., 1551, "Carta a Antonio Agustín» fechada el 26 de abril de 1551 (Ms. RAH 9-6002-9.ª, copia del siglo Xviri del original del Vat. Lat. 6040, 1503-212v.

Gimeno Pascual, H., 1997, Historia de la investigación epigráfica en España en los siglos XVI-XVII a la luz del recuperado manuscrito del conde de Guimera, Zaragoza.

González Herrero, M., 2002, «M. Fidius Fidi f. Quir(ina) Macer, benefactor de Capera», Gerión 20, 417433.

—, 2004, "Prosopografía de praefecti fabrum originarios de Lusitania», Revista portuguesa de Arqueología vol. 7 n. ${ }^{\circ} 1,365-384$.

González Palencia, Á., 1932, Don Luis de Zúñiga y Ávila. Gentilhombre de Carlos V, Madrid.

Gruterus, J., 1602, Inscriptiones Antiquae Totius Orbis Romani in corpus absolutiss(imum) redactae cum indicib(us) XXIV, Heidelberg.

Guerra y Cumbreño, F.L., 1865, Notas a las antigüedades de D. José de Viu, Cáceres.

Нава Quirós, S., 1986, Catálogo epigráfico del partido judicial de Plasencia, Memoria de Licenciatura leída en la Facultad de Filosofía y Letras de la UEX en septiembre de 1986, Cáceres.

HüBner, E., 1869, Corpus Inscriptionum Latinarum II, Inscriptiones Hispaniae Latinae [CIL II], Berolini.

Hurtado de San Antonio, R., 1977 Corpus provincial de inscripciones latinas. Cáceres, Cáceres.

Mameranus, N., 1533-1535, Epitaphia et Antiquitates Romanorum per Hispaniam. Ms. Del siglo Xvi del que se conservan dos copias, el llamado Codex Augustanus en la Bibliotheca Augustana, Ausburg, sign. 656) y un segundo ejemplar en la Niederssachische Landesbibliothek de Hannover, sign. XXVIII 1653.

Marcks-Jacobs, C., 2001, «Die Antikensammlung des D. Luis de Ávila y Zúñiga, Marqués de Mirabel, in Plasencia, mit 2 Textabbildungen uns Tafel 18-34», Madrider Mitteilungen 42, 155-208.

Mayans y Siscar, G., 1746, Fancisci Sanctii Brocensis, in inclyta Salmanticensi Academia Emeriti, olim Rhetorices, et Primarii Latinae, Graecae Linguae Doctoris, Opera Omnia, una cum eiusdem scriptoris vita, vol. 1, Geneva.

Mélida Alinari, J.R., 1924, Catálogo monumental de España. Provincia de Cáceres. 1914-1916, Madrid.

Méndez, F., 1771, Noticias de la vida y escritos del Rmo. P. Mro. Fr. Henrique Florez de la orden del Gran P. S. Agustin, cathedratico de la Universidad de Alcalá, y Asistente General de las Provincias de España. Con una relación individual de los Viages que hizo à las Provincias y Ciudades mas principales de España, Madrid.

Mommsem, T., 1878, «De fide Leonhardi Gutensteni», Ephemeris Epigraphica 1, pp. 67-77.

Morales y Tercero, A., 1753, Inscripciones y estatuas de Plasencia (Ms. RAH 9-5676-5).

Muratori, L.A., 1740, Novus thesaurus veterum inscriptionum in praecipuis earumdem collectionibus hactenus praetermissarum, vol. 3, Mediolani.

Navarro Caballero, M., J.L. Ramírez Sádaba (coord.), 2003, Atlas antroponímico de la Lusitania romana, Mérida.

Nünnerich-Asmus, A., 1996, El arco cuadrifonte de Cáparra (Cáceres). Un estudio sobre la arquitectura Flavia en la Peninsula Ibérica, Anejos de AEspA XVI, Madrid.

Occo, A., 1596, Inscriptiones veteres in Hispania repertae, Heidelbelrg.

Osório, M., 2006, «Placa funeraria de Aldeia de Santa Madalena (Guarda)», Ficheiro Epigráfico 82, 6-9, n. ${ }^{\circ} 366$. 
Ponz Piquer, A., 1784, Viaje de España; en que se da noticia de las cosas más apreciables y dignas de saberse, que hay en ella, t. VII, carta quinta, Madrid.

Rodríguez Moñino, A., 1931, Una visita de archivo en el siglo diez y ocho (Ascensio de Morales en Plasencia. 1753 (Cartas), Badajoz.

SÁnchez Paredes, A., 1976, "Inscripciones latinas de la Vettonia», V Coloquios de Estudios Extremeños, Badajoz, 85-98.

Sánchez Loro, D., 1982, Historias placentinas inéditas, vol. A, Cáceres.

Sayans Castaños, M., 1961, Descripción de la ciudad y obispado de Plasencia por Luis de Toro, físico y médico de Plasencia del siglo XVI, Plasencia.

Tornay Cabrera, M., 2012-2013, "El Señorío de Galisteo y los III Condes de Osorno: Un ejemplo de Mecenazgo renacentista", Norba 32-33, 25-40.

Vallejo Ruiz, J.M., 2005, Antroponimia indigena de la Lusitania romana, Vitoria-Gasteiz.

VANnÉRus, J., 1949, "Mameranus et sa famille», en Bibliographie nationale du Pays de Luxembourg depuis ses origines jusq'á nos jours (collectión présentée pour Julies Mersch), fac. II, Lusembourg, 299-321.

VIU, J., 1852, Estremadura. Colección de sus inscripciones y monumentos seguida de reflexiones importantes sobre lo pasado, lo presente y el porvenir de estas provincias, Imprenta de Pedro Montero, Madrid.

—, 1864, Colección de Inscripciones y Antigüedades de Estremadura, Cáceres. 